\title{
Multimodal compression of 3D hyperspectral images using spiral insertion technique and JPEG2000
}

\author{
Akrour leila $^{1}$, Lahdir Mourad ${ }^{2}$, Nait Ali Amine ${ }^{3}$, Ameur Soltane $^{4}$ \\ ${ }^{1,2,4}$ (Laboratoire d'Analyse et Modélisation des Phénomènes Aléatoires (LAMPA), Department of Electronics, \\ Mouloud Mammeri University (UMMTO), Po. Box 17 RP 15000, Tizi-Ouzou, Algeria, \\ ${ }^{3}$ (Laboratoire Images, Signaux et Systèmes Intelligents (LISSI), Université Paris-Est Créteil, Lissi, EA. 3956, \\ 61, avenue du Général de Gaulle 94010, Créteil France)
}

\begin{abstract}
We introduce in this paper a new scheme of multimodal hyperspectral images compression based on the spiral insertion technique. The approach in question is to interleave the image pixels less rich in information of hyperspectral sequence in other images of the same sequence allowing minimizing the rate of hyperspectral data to compress. This spiral insertion is performed edges of the images, can benefit the central region of the sequence. And that represents the interest region (ROI) to preserve during compression. The reduced sequence called multimodal is compressed efficiently by the JPEG2000 adapted to 3D discrete wavelet decomposition. After decompression, the images that are evaluated by objective criteria are faithfully reproduced and the memory space required for their storage and transmission is reduced.
\end{abstract}

Keywords: Compression, hyperspectral, multimodal, spiral insertion, ROI, JPEG2000

\section{INTRODUCTION}

For processing and storing the growing volume of data from hyperspectral image sensors embedded in the new generation of satellites, the development of compression algorithms fit to exploiting the spatial and spectral redundancy of this data is necessary. The term hyperspectral is generally used for spectral data containing hundreds of sample spectra [1]. Hyperspectral images present thus specific characteristics which require to be exploited by an efficient compression algorithm. We used in this article a database of 36 hyperspectral images from AVIRIS sensor downloaded on the NASA website. We have used a dataset of the Yellowstone scene, acquired in 2006 and having a size of 512 x 614 over 224 optical bands. This AVIRIS calibrated radiance images can be downloaded from http://aviris.jpl.nasa.gov/html/aviris.freedata.html. By their nature three-dimensional data, hyperspectral images are similar to video data and certain medical data [2] [3] [4] [5]. Compressions of such data pose a challenge to the data compression community [1] [6] [7] [8]. For compression of hyperspectral images, two major systems exist: compression systems-oriented applications such as classification and generic type systems [7] [9] [10]. Compression systems oriented classifications are based on vector quantization with creation of a dictionary (codebook) [11]. Generics systems are based on a classic scheme of transform compression [12]. Various studies have been conducted with different transforms, especially in spectral level: using a Karhunen-Loeve (also called Principal Component Analysis), a DCT, an independent component analysis (ICA) and wavelet [10] [11] [13]. The wavelet transform among the most widely used. In fact, wavelets showed good efficiency on various data while maintaining a reasonable complexity [14]. The JPEG2000 standard is one of the methods that take advantage of the pixels decorrelation after wavelet transform [8] [15]. In this paper, we propose a new approach to pre-process 3D hyperspectral images before using any compression scheme. The idea consists in compacting (i.e. reducing the number of images of a sequence to compress) any volume/sequence in a context of Multimodal Compression based on the concept introduced in [16] related to image-signal merging and video-signal merging of biomedical data. The scheme presented in this work is considered as a variant and an extension since it deals with 3D images. Generally speaking, the idea of Multimodal Compression consists in merging data using an insertion function into an image or a set of images, before the encoding process. Afterwards, a separation function is used to extract the required information from the decoded data. The multimodal scheme is based on the spiral technique insertion. In this technique, the pixels of the poorer images in information of the hyperspectral sequence are interlaced in the other images in the same sequence, to minimizing the rate of hyperspectral data to compress. This spiral insertion that performed at the edges of the image can benefit the central region of the image sequence. This latter represents the region of interest (ROI) to preserve during compression. The reduced sequence called multimodal is compressed efficiently by JPEG2000. Consequently, a fast encoding/decoding is achieved without any significant loss of information. This paper is organized as follows: in Section II, the methodology of Multimodal Compression extended to hyperspectral volumetric data, is presented. Results and performance analysis evaluated on AVIRIS database are presented in section III. Finally, a conclusion is 
provided in section IV.

\section{METHODOLOGY}

Consider a hyperspectral sequence seq (i) to be compressed, wherein the position of an image is identified by $\mathrm{i}$ ( $\mathrm{i}$ is from 1 to 36). The Multimodal Compression of this sequence requires various phases: analysis, insertion and encoding (see figure 1). In a first step, the number of images constituting the hyperspectral sequence seq (i) is reduced by eliminating those with a smaller information rate. The pixels of these are inserted in a spiral by replacing a pixel in tow at the edge of the remaining images. The central region which forms a ROI for the compression phase is left without sampling. In a second step, the reduced sequence deduced seqr ( $\mathrm{j}$ ) (with $\mathrm{j}$ is from 1 to $\mathrm{M}<36$ ) which includes all the information of the initial sequence is efficiently compressed by JPEG2000 to generate a binary sequence containing useful information to save. The decompression is then performed by proceeding by inverse transformations.

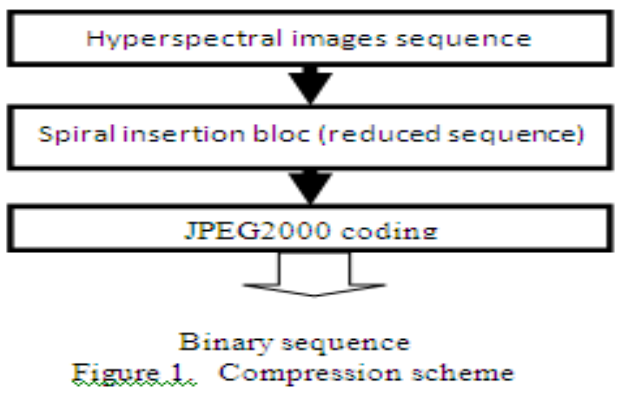

III. ANALYSIS PHASE

In this phase, hyperspectral images to be compressed are sorted so that those containing less information are considered potentially appropriate to be merged in the remaining images. For this purpose, an objective criterion should be defined. This criterion can perform a simple statistical analysis along the image channels. In this work, we consider that the smooth images are relatively poor in terms of information. Therefore, the variance and energy are used as indicator to sort images from the highest variance to the lowest one. The initial hyperspectral sequence seq (i) is, firstly, statistically studied, by calculating for each of the channels the statistical parameters. Statistical analysis of pixels characterizing each image of the sequence is essential for the development of the spiral insertion technique described in this article. Indeed, this analysis aims to establish criteria for choosing the number and images to eliminate to form the multimodal sequence to compress seqr (j). Variance (Var) and energy (E) were calculated for the sequence of 36 images (channels) extracted from the AVIRIS database. The figure 2 shows the evolution of energy and variance parameters of the sequence seq (i). The variance of the coefficients of the superior's channels is much greater than that of the lower channels. As a result, the first channels of the sequence have a better compression rate / distortion. The energy values show a greater concentration of information for higher channels.

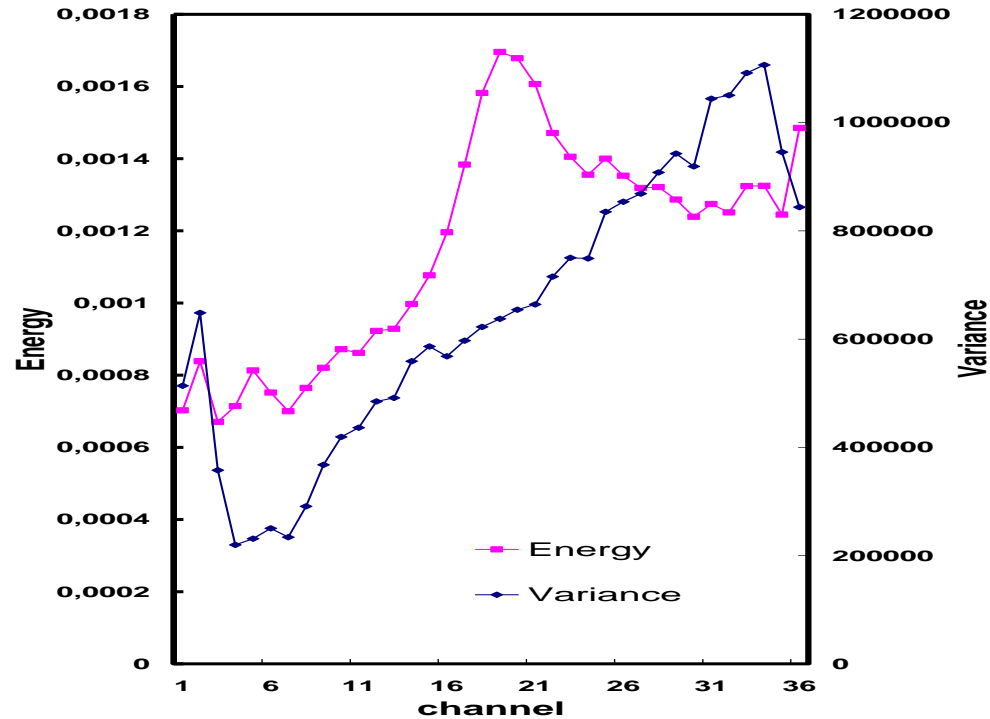

Figure 1. The variance and Energy evolutions of the hyperspectral sequence 


\section{MIXING PHASE}

Given all the properties thus put in evidence and to optimize the compression phase, the hyperspectral sequence seq (i) is reduced. A classification based on the information rate carried by each of the images constituting the sequence is established. The first images of the sequence are inserted into the other remaining images. For each remaining image in the sequence of dimension (ht, wd), a filling zone $\mathrm{Z}$ at its edge is determined (see figure 3). $\mathrm{Z}$ varies according to a parameter $\mathrm{P}$, which shows the degree of filling. These two parameters are related by the following equation:

$$
\mathrm{Z}=\left(\frac{h t / 2}{P}\right)
$$

The region of interest ROI is the zone of interest will be preserved during the compression step. The filling operation is to interleave the pixels of the images to eliminate in the sequence by replacing one pixel in two on each of the remaining images. Note that the number of images that are to be sampled for each of the images to eliminate varies with distance from the filling region $\mathrm{Z}$. After the sampling step, the hyperspectral sequence is thus reduced, constituting a new sequence called multimodal seqr (j), whose the number of images has been reduced, but containing the maximum energy of information of the initial sequence. The images of the multimodal sequence seqr (j) thus formed are coded efficiently by JPEG2000.

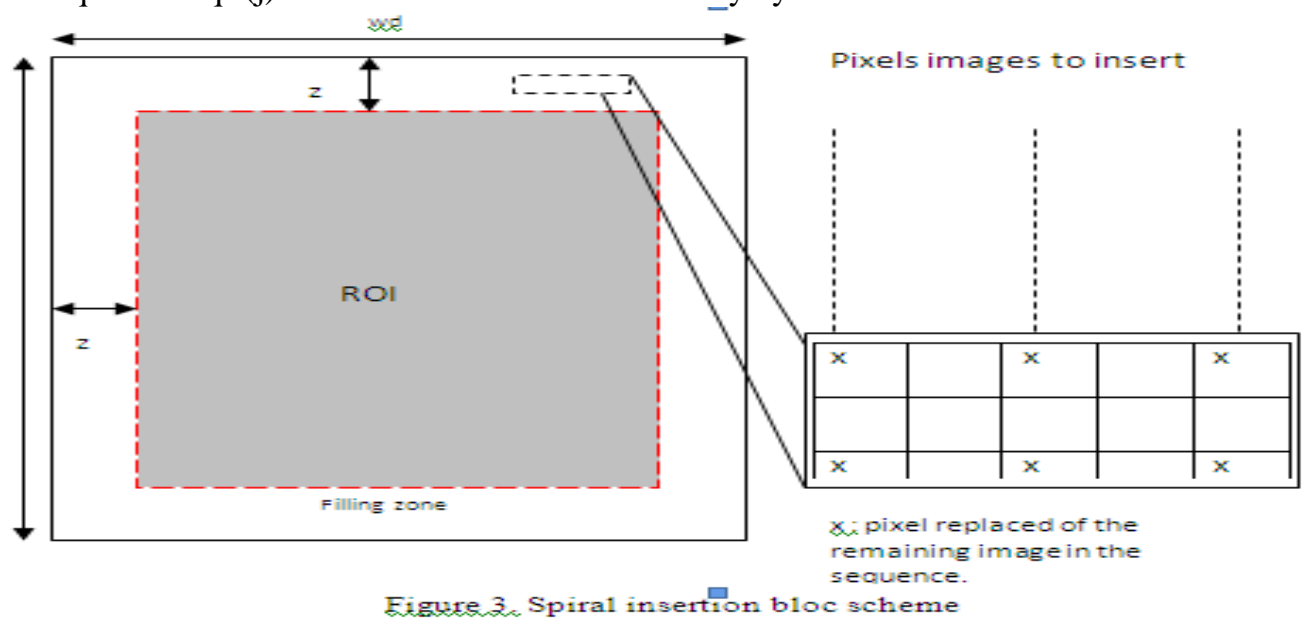

\section{DECODING PHASE}

The decompression process (decoding of the bit sequence) permit to reconstruct the sequence seqr (j) (see figure 4). At this phase, a reduced volume containing the data mixture is obtained.

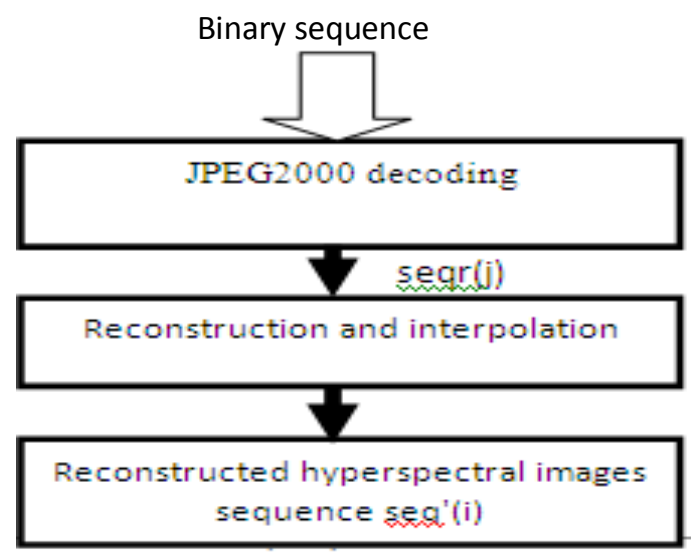

Figure 2. Decompression scheme

VI. SEPARATION PHASE

After the decoding phase, this step consists in extracting the embedded images from the contours of each image corresponding to a decoded reduced volume. Afterwards, an interpolation is performed on the 
contours in order to estimate missing pixels values. The interpolation used here is linear. It is calculated from the neighbourhood of each missing pixel. Hence, the interpoled pixel $p_{i}(m, n)$ is given by:

$$
p_{i}(m, n)=\frac{1}{4} \sum_{l=1}^{2}\left(p\left(m, n+(-1)^{l}\right)+p\left(m+(-1)^{l}, n\right)\right)
$$

where $(m, n)$ defines the interpoled pixel position.

After this operation, a new sequence of images seq'(i) is reconstituted representing an approximation of the original sequence.

\section{SIMULATIONS RESULTS}

To appreciate the relevance of the results obtained, two characteristic were considered. The compression rate (bpp) and peak signal to noise ratio (PSNR). The latter being expressed in peak values, is determined by comparing the reconstructed image after compression / decompression to the original image. This quantity is defined by:

$$
P S N R=10 \log \left(\frac{\left(2^{b}-1\right)^{2}}{M S E}\right)
$$

In this equation, MSE is the mean squared error calculated by considering the differences between the original image and the reconstructed image (coding on b bits). Compressing the hyperspectral sequence seq (i) is reduced to the multimodal sequence form seqr (j). Setting the fill area to $20 \%$, four images are inserted in the remaining images. After this reduction, the multimodal sequence is then only composed from 32 images. We give below the results of our compression algorithm. We varied at maximum the bit rate per pixel (bpp) while using as the endpoint PSNRM calculated between the 32 images based multimodal. This ratio is defined by:

$$
\operatorname{PSNRM}=\frac{1}{32} \sum_{i=1}^{i=32} P S N R(i)
$$

The curves in figure 5 show the variations in the rate PSNRM bpp for the original sequences (seq) and reduced (seqr). Based on these curves, we can see that for low rates, the difference in performance in terms of PSNR with and without insertion spiral is obviously minimal. This difference is even at very small region of interest (ROI). This result is particularly interesting because in these conditions, we can ensure that our approach to insertion spiral has a double advantage. On the one hand, it does not degrade the visual quality of reconstructed images in the sequence and secondly, the computation time of our algorithm applied to multimodal sequence is the same size as the original sequence. In addition, the execution time required for insertion steps and interpolation are very small.

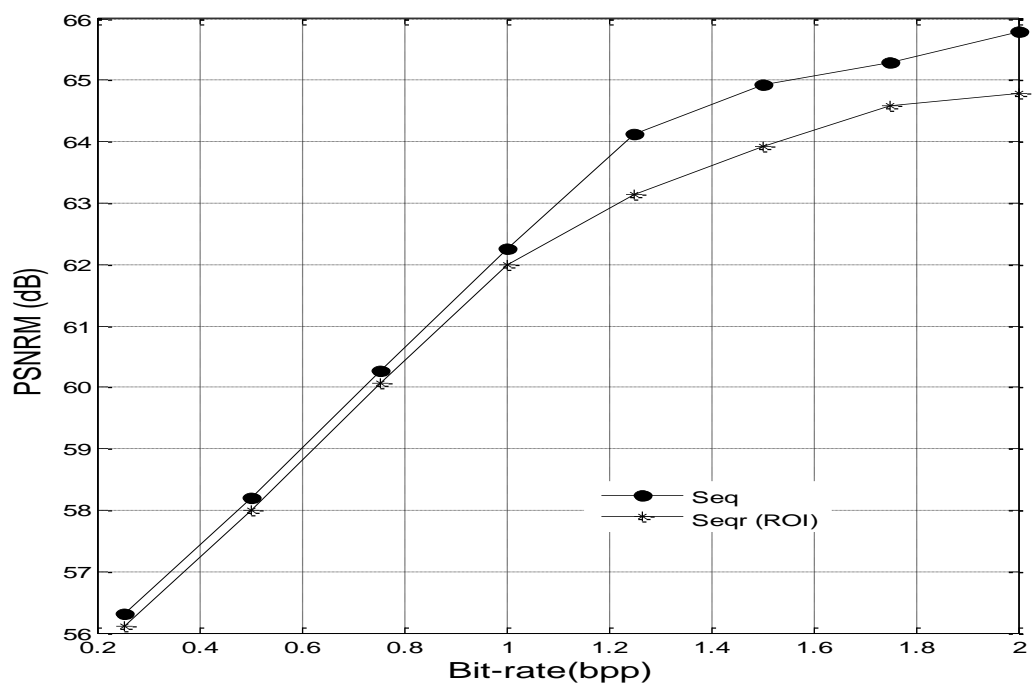

Figure 3. Evolution du PSNRM en fonction du bpp 


\section{CONCLUSION}

A compression algorithm for hyperspectral images using spiral insertion technique is presented in this article. The approach is indeed to insert the pixels of hyperspectral images in other images taken in the same sequence, which helped to minimize the amount of data to compress hyperspectral very important. Sequence deduced called multimodal has been compressed efficiently by the JPEG2000 encoder. The results obtained on a sequence AVIRIS hyperspectral images have shown that for low flow, compress the sequence is reduced multimodal advantageous in terms of performance (speed, distortion, and computation time) to compress the original sequence. These results are preliminary recesses, but very encouraging for the use of the technique of inserting spiral compression of satellite images. Also, the compression of hyperspectral images based on the JPEG2000 encoder yielded very low rates for uncompressed images of good visual quality without distortion.

\section{ACKNOWLEDGEMENTS}

Corresponding Author is thankful to all reviewers and Authors whose references are taken to prepare this article.

\section{REFERENCES}

[1]. E. Christophe, C. Mailhes, and P. Duhamel, Hyperspectral image compression: Adapting SPIHT and EZW to anisotropic 3D wavelet coding, IEEE Transactions on Image Processing, 17(12), 2008, 23342346.

[2]. P. Raffy, Y. Gaudeau, D. Miller, J-M. Moureaux and R.A Castellino, Effect of 3D Wavelet Image Compression on Computer Aided Detection (CAD) Lung Nodule Volumetry, Academic Radiology, 13(10), 2006, 1194-1203.

[3]. H. Benoit-Cattin, A. Baskurt and R. Prost, 3D medical image coding using separable 3D wavelet decomposition and lattice vector quantization, Signal Processing, 59, 1997, 139-153.

[4]. S. Cho, D. Kim and W.A Pearlman, Lossless Compression of Volumetric Medical Images with Improved Three-Dimensional SPIHT Algorithm, Journal of Digital Imaging, 17, 2004, 57-63.

[5]. B.J. Kim and W.A. Pearlman, An Embedded Wavelet Video Coder Using Three-Dimensional Set Partitioning in Hierarchical Trees (SPIHT), In IEEE Data Compression Conference DCC'97, 1997, 221260.

[6]. P. L. Dragotti, P. Giovanni, and A. R. P. Ragozini, Compression of multispectral images by three dimensional SPIHT algorithm, IEEE Trans. On Geoscience and Remote Sensing, 38, 2000, 416-428.

[7]. T. W. Fry, Hyperspectral Image Compression on Reconfigurable Platforms, Master Thesis, University of Washington, Seattle, Washington, 2001.

[8]. J. T. Rucker, J. E. Fowler, and N. H. Younan, JPEG2000 coding strategies for hyperspectral data, in Proceedings of the International Geoscience and Remote Sensing Symposium, 1, Seoul, South Korea, July 2005, 128-131.

[9]. X. Tang and W. A. Pearlman, Three-Dimensional Wavelet-Based Compression of Hyperspectral Images, chapter in Hyperspectral Data Compression, (Kluwer Academic Publishers. Available on http://www.cipr.rpi.edu/ Pearlman, 2005).

[10]. Q. Du et J. E. Fowler, Hyperspectral image compression using JPEG2000 and principal component analysis, IEEE Geoscience and Remote Sensing Letters, 4(2), 2007,201-205.

[11]. S. Ameur, A. Adane et M. Lahdir. Compression d'images Météosat en sous bandes par transformation discrète en cosinus et quantification vectorielle. Télédétection, 2(4), 2002, 255-266.

[12]. B. Penna, T. Tillo, E. Magli et G. Olmo, Transform coding techniques for lossy hyperspectral data compression, IEEE Trans. Geoscience and Remote Sensing, 45(5), 2007, 1408-1421.

[13]. M. Lahdir, S. Ameur et A. Adane. Algorithme non itératif basés sur les ondelettes biorthogonales et les fractales pour la compression des images satellitaires. Télédétection, 6(4), 2006, 345-360.

[14]. M. Antonini., M. Barlaud, P. Mathieu and I. Daubechies, Image Coding using wavelet transform, IEEE Trans. On Image Processing, 1(2), 1992, 205-220.

[15]. S. Taubman et M.W. Marcellin, JPEG2000 Image compression fundamentals, standards and practice, (Kluwer Academic, 2002).

[16]. A. Naït-Ali, E H Zeybek, and X. Drouot, Introduction to Multimodal Compression of Biomedical data. In the book: Advanced Biosignal Processing. (Springer, 2009), 353-375. 